\title{
Validade e reprodutibilidade do Teste Avaliativo de DTM: um estudo de acurácia diagnóstica
}

\author{
Validity and reproducibility of the TMD \\ Assessment Test: diagnostic accuracy study
}

\author{
Yasmin Bianca Oliveira Neri' ${ }^{1}$ (1) \\ Ana Carolina Silva Francisco 2 (1) \\ Isabela Raul Nepomuceno de Jesus ${ }^{3}$ (1)
}

\author{
Italo Eduardo Novaes Santos ${ }^{4}$ (1) \\ Rafael Santos Ferreira 5 \\ Ana Lúcia Barbosa Góes ${ }^{6}$ (1)
}

\begin{abstract}
${ }^{1}$ Autora para correspondência. Universidade Federal da Bahia (Salvador). Bahia, Brasil. yasmineri6@gmail.com
2-5Universidade Federal da Bahia (Salvador). Bahia, Brasil. anacarollinafisio@outlook.com, isabelaraulndj@gmail.com, italoeduardo03@yahoo.com rsferreirars@hotmail.com

שUniversidade Federal da Bahia (Salvador); Escola Bahiana de Medicina e Saúde Pública (Salvador). Bahia, Brasil. goes.ana@ufba.br
\end{abstract}

RESUMO | INTRODUÇÃO: Disfunções temporomandibulares (DTM) são distúrbios na ATM, sendo de origem muscular, articular ou mista, e com íntima relação com alterações posturais. Instrumentos de diagnóstico apresentam lacunas quanto à aplicação clínica e não associam à postura. OBJETIVO: Validar o Teste Avaliativo de DTM (TAvDTM) quanto à acurácia diagnóstica e reprodutibilidade. MATERIAIS E MÉTODOS: Estudo de acurácia diagnóstica com indivíduos entre 18 e 59 anos avaliados pelo Índice Anamnésico de Fonseca (IAF), questionário sociodemográfico e TAvDTM, este último realizado por três examinadores diferentes e treinados. O resultado da categorização do diagnóstico do IAF foi comparado com o resultado do TAvDTM. Para acurácia diagnóstica utilizou-se teste Qui-Quadrado. Valores Preditivos Positivos (VPP) e Negativos (VPN) foram determinados. A reprodutibilidade entre os três examinadores foi feita por meio do Kappa de Cohen, para análise $2 \times 2$ e de Fleiss. Todos os testes com significância de 5\%. RESULTADOS: Dos 10 participantes avaliados, o IAF identificou 8 com diagnóstico de DTM, enquanto o TAvDTM verificou 9 participantes com presença desta disfunção. A sensibilidade foi de $100 \%$, especificidade de $50 \%$, VPP de $88 \%$ e VPN de $50 \%$. O Kappa de Fleiss evidenciou confiabilidade razoável ( $K=0,26$ [IC 95\%: -0,099 - 0,617]; $p>0,05)$. O Kappa de Cohen mostrou reprodutibilidade insignificante entre os avaliadores 1 e $2(K=-0,11 ; p>0,05$; discordância $=80 \%)$, e 1 e $3(K=-0,11 ; p>0,05$; discordância $=80 \%)$, reprodutibilidade perfeita entre os avaliadores 2 e 3 ( $K=1,00$; $p<0,05$; concordância=100\%). CONCLUSÃO: O TAvDTM apresenta alta sensibilidade e baixa especificidade, porém com baixa capacidade de reprodução até o presente momento.

PALAVRAS-CHAVE:TranstornosdaArticulaçãoTemporomandibular. Inquéritos e questionários. Acurácia diagnóstica. Reprodutibilidade dos testes.
ABSTRACT I INTRODUCTION: Temporomandibular disorders are TMJ disorders of muscular, articular, or mixed origin and closely related to postural alterations. Diagnostic tools have gaps regarding clinical application and do not associate posture. OBJECTIVE: Validate the TMD Assessment Test (TMDAsT) regarding its diagnostic accuracy and reproducibility. MATERIALS AND METHODS: Diagnostic accuracy study with individuals between 18 and 59 years old evaluated by the Fonseca Assessment Index (FAl), sociodemographic questionnaire, and TMDAsT, the latter performed by three different trained examiners. The result of FAI diagnosis categorization was compared with the result of TMDAsT. A Chi-square test was used for diagnostic accuracy. Positive predictive values (PPV) and negative predictive values (NPV) were determined. The Kappa of Fleiss did the reproducibility between three examiners. Cohen's Kappa, for $2 \times 2$ analysis. All tests with $5 \%$ significance. RESULTS: Of the 10 participants assessed, FAI identified 8 participants with a TMD diagnosis while the TMDAsT verified 9 participants with this dysfunction. Sensitivity was $100 \%$, specificity 50\%, PPV $88 \%$ and NPV 50\%. Fleiss' Kappa showed reasonable reliability ( $\mathrm{K}=0.26$ [95\% Cl: $-0.099-0.617] ; \mathrm{p}>0.05)$. Cohen's Kappa showed insignificant reproducibility between observers 1 and 2 $(\mathrm{K}=-0.11 ; \mathrm{p}>0.05$; discordance $=80 \%)$, and 1 and $3(\mathrm{~K}=-0.11 ; \mathrm{p}>0.05$; discordance $=80 \%$ ), perfect reproducibility between observers 2 and $3(\mathrm{~K}=1.00 ; \mathrm{p}<0.05$; concordance $=100 \%)$. CONCLUSION: TMDAsT presents high sensitivity and low specificity but with low reproducibility until the present moment.

KEYWORDS: Temporomandibular joint disorders. Survey and questionnaires. Diagnostic accuracy. Reproducibility of results. 


\section{Introdução}

As disfunções temporomandibulares (DTM) são definidas como um conjunto de desordens articulares e/ou musculares que levam a dificuldade no funcionamento da articulação temporomandibular (ATM). Apresenta etiologia multifatorial e tem associação com traumas (mandibulares e na ATM), hábitos parafuncionais (mascar chicletes, morder lábios e bochechas, roer objetos, apertar ou ranger os dentes), desvios posturais, entre outros. ${ }^{1}$

Frequentemente, a DTM é diagnosticada na população entre 19 e 40 anos.? Esse transtorno apresenta uma incidência na população mundial, na qual $65 \%$ manifesta, no mínimo, um sintoma, e 35\% um sinal dessa disfunção ${ }^{3}$, tendo prevalência no gênero feminino ${ }^{4}$, que tem a maior probabilidade de apresentar sintomas, como dor no pescoço e ombros, nos músculos faciais, na articulação temporomandibular e cefaleia.?

Quanto à postura, a severidade dessas alterações apresenta uma relação com a flexibilidade da cadeia muscular posterior.. Os desvios posturais de coluna cervical, cabeça, ombros e outros segmentos podem ser fatores causais de disfunção craniocervical, provocando posteriormente a perpetuação de sintomas e sinais de DTM., 2,, 7 Também foi identificado que os músculos responsáveis pela mastigação têm relação com a postura corporal, por meio de complexas conexões neuromusculares. Com isso, entende-se que existe relação entre alterações na ATM e alinhamento postural.,.,

Considerando postura como uma habilidade complexa baseada na interação de processos sensóriomotores dinâmicos e entendendo que a relação das peças ósseas depende da interação das diversas cadeias musculares ${ }^{8}$, é possível considerar a existência da associação entre a DTM e cadeias musculares, que são músculos que trabalham em sinergia para a manutenção da postura e do funcionamento adequado das estruturas corporais. Com isso, se uma área está comprometida pode afetar outras regiões relacionadas por essas cadeias musculares.

$\mathrm{Na}$ literatura, existem diversos instrumentos-12 comumente adotados na área de fisioterapia e odontologia com o intuito de obter um correto diagnóstico de DTM e assim oferecer o tratamento mais apropriado. Dessa forma, foi desenvolvido o Research Diagnostic Criteria for Temporomandibular Disorders (RDC/TMD), que é um dos questionários mais utilizados em ambientes clínicos e em pesquisa, desde a sua publicação em 1992. Entretanto, assim como outros instrumentos, esse questionário apresenta lacunas como dificuldades de aplicação na prática clínica, por ser um questionário voltado para a pesquisa, bastante complexo e que apresenta um longo tempo de aplicação. 11

Como a exemplo de ferramentas de fácil administração, temos o Diagnostic Criteria for Temporomandibular Disorders (DC/TMD), que é utilizado tanto para pesquisa quanto para prática clínica, sendo atualmente considerado como padrão ouro. Além dele, também há o Índice Anamnésico de Fonseca (IAF), que é empregado para determinar a severidade da DTM de acordo com seus sinais e sintomas, sendo amplamente utilizado como ferramenta de triagem, desde a sua validação. Contudo, esses índices não consideram a postura como uma variável a ser incluída na avaliação. $\frac{13}{}$

É notória a crescente relação existente entre postura e disfunção temporomandibular apresentada na literatura ${ }^{6}$; assim, foi proposto o Teste Avaliativo de Disfunção Temporomandibular, composto por cinco movimentos posturais (flexão de tronco, inclinação lateral à direita, inclinação lateral à esquerda, torção lateral à direita e torção lateral à esquerda), em que o avaliador realiza esses movimentos de forma passiva e analisando a presença de limitações musculares. Esse teste leva em consideração a análise da tensão da cadeia muscular posterior, que possui a finalidade de identificar indivíduos com ou sem disfunção temporomandibular.

Por se tratar de um teste não validado na literatura, se faz necessária a sua validação antes de implementá-lo na prática clínica ou em ambientes de pesquisa. Com isso, o objetivo deste estudo foi validar o Teste Avaliativo de Disfunção Temporomandibular quanto à sua acurácia diagnóstica e possibilitar aos profissionais de saúde uma ferramenta validada que, além de complementar no diagnóstico da presença da DTM, permite a relação com alterações posturais, possibilitando que os profissionais de saúde ofereçam um tratamento adequado à população. 


\section{Métodos}

Foi realizado um estudo de acurácia diagnóstica, cego e de caráter prospectivo, no período de outubro de 2019 a março de 2020. Todos os participantes da pesquisa assinaram o Termo de Consentimento Livre e Esclarecido (TCLE), de acordo com a resolução $n^{\circ}$ 466/12 do Conselho Nacional de Saúde. Essa pesquisa foi aprovada pelo CEP ICS-UFBA sob o CAAE de $\mathrm{n}^{\circ} 26305519.4 .0000 .5662$

Esse estudo foi realizado com o intuito de identificar o alinhamento entre os examinadores da pesquisa para melhor aplicação do Teste Avaliativo de Disfunção temporomandibular já que se trata de um instrumento avaliador-dependente.

Os critérios de inclusão utilizados foram indivíduos de ambos os sexos com idade entre 18 e 59 anos. Como critérios de exclusão, indivíduos com acidente vascular encefálico ou que realizaram cirurgia bucomaxilar ou ainda indivíduos que apresentaram traumatismo de face, presença de artrite reumatoide, diagnóstico de escoliose, degeneração de quadril e coluna, que realizaram tratamento fisioterapêutico ou ortodôntico para a disfunção temporomandibular, que apresentassem perda dentária ou estivessem em uso de medicamentos (analgésicos, anti-inflamatórios e relaxantes musculares).

Inicialmente, um avaliador aplicou um questionário sociodemográfico; em seguida, foi utilizado o IAF como padrão ouro na comparação entre os testes. Esse índice possui dez itens com possibilidade de respostas: sim (10 pontos), às vezes (5 pontos) e não (0 pontos), sendo a sua classificação definida em: sem DTM (0 a 15 pontos), DTM leve (20 a 45 pontos), DTM moderada (50 a 65) e DTM severa (70 a 100 pontos). Não foi possível utilizar o DC/TMD, atual padrão-ouro para diagnóstico de DTM, porque este ainda estava em processo de validação no período da coleta desse estudo.

Após a administração dos testes, os participantes foram analisados com o Teste Avaliativo de DTM, em ordem aleatória, por três avaliadores devidamente treinados e as avaliações ocorreram com intervalo de 15 minutos entre os avaliadores para evitar estímulos musculares e não causar interferência nos resultados.
Segundo a filosofia das cadeias musculares de Busquet, os movimentos da cadeia posterior indicam a relação existente entre postura e DTM, enquanto a cadeia muscular anterior indica relação com disfunção de órgãos ou com disfunção visual.

Para a realização do Teste Avaliativo de DTM, a pesquisa contou com um avaliador, que tinha a função de controlar os examinadores; três examinadores, que aplicaram os padrões de movimentos que causam tensionamento da cadeia muscular posterior e que determinam a presença ou não de DTM. O diagnóstico possível para o instrumento seria negativo, uma cruz (+) ou duas cruzes (++). Aqueles que receberam a partir de $(++)$ em qualquer padrão de movimento foram diagnosticados com disfunção temporomandibular, e os indivíduos que receberam (+) ou negativo foram considerados sem a presença de DTM, sendo $(+)$ indicativo de leve resistência muscular aos movimentos realizados, não interferindo no diagnóstico.

Importante salientar que os participantes foram identificados a partir do número de registro, garantindo anonimato, e que os examinadores estavam cegos entre eles, já que cada um se encontrava em uma sala isolada, não tendo contato de um examinador com o outro.

As instruções para a realização do teste foram: 1 - O indivíduo em posição de ortostase, com os pés juntos, descalço, com a postura relaxada e olhar voltado para frente; 2 - Os movimentos foram realizados apenas pelo examinador, ou seja, de forma passiva e caso o indivíduo tentasse ajudar no movimento, esse seria interrompido e começaria da postura inicial. O movimento foi repetido quantas vezes fossem necessárias para o diagnóstico; 3- Se o indivíduo fizesse uso de óculos, deveria permanecer utilizando-o durante a execução do teste.

Para realização do Teste avaliativo de DTM (figura 1) o examinador observou as tensões musculares posteriores produzidas pelos seguintes movimentos:

A- Flexão do tronco ou teste das tensões posteriores - que consistiu em promover enrolamento de todo o corpo do participante, começando pela cabeça e progredindo lenta e suavemente por toda a coluna. 
B- Inclinações laterais direita e esquerda do tronco ou teste das tensões laterais - permite inclinação lateral de todo o corpo do participante, começando pela cabeça e progredindo lenta e suavemente por toda a coluna.

C- Torção anterior direita e torção anterior esquerda do tronco ou teste das tensões oblíquas posteriores - promove torção anterior de todo o tronco do participante de forma lenta e suave.

Na presença de intensa resistência em qualquer desses movimentos, o teste deveria ser parado imediatamente.

Figura 1. Posturas realizadas durante a aplicação do Teste Avaliativo de Disfunção Temporomandibular.
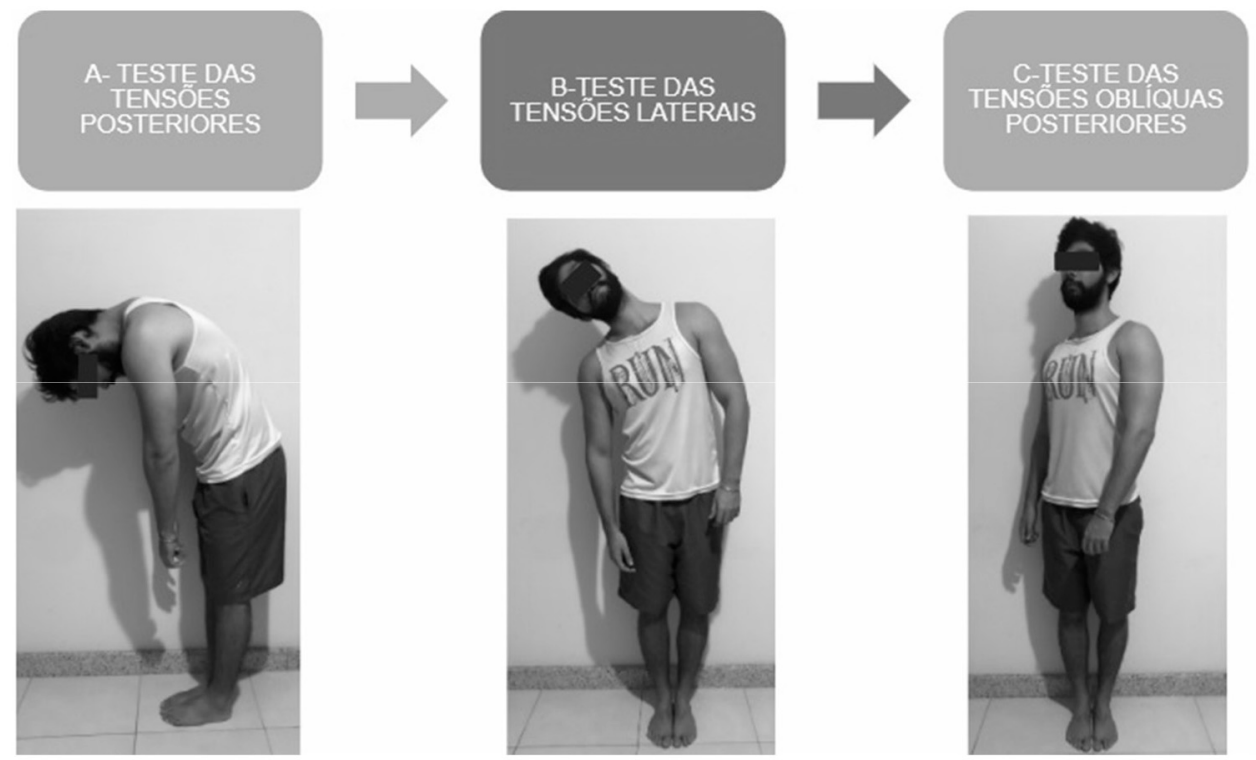

Todos os dados avaliados foram armazenados em planilhas excel, v2109, para posterior análise. As análises estatísticas foram realizadas pelo Software SPSS, versão 23.0. Para fins de comparação com os resultados do Teste Avaliativo de DTM, este estudo recategorizou os resultados do IAF, uma vez que este oferece quatro possibilidades de resposta e o teste avaliativo tem desfecho dicotômico. A resposta sem DTM no IAF se manteve a mesma na recategorizaçã, e todas as outras possibilidades (DTM leve, moderada e severa) foram recategorizadas como "Presença de DTM".

Para analisar a sensibilidade (\% de indivíduos com a presença de DTM) e especificidade (\% indivíduos sem a presença de DTM) com relação ao Índice Anamnésico de Fonseca, foi realizado o teste do Qui-Quadrado, solicitando o percentual de combinação do Teste Avaliativo em relação ao IAF. Também foi feita análise de Valores preditivos positivos (VPP) e valores preditivos negativos (VPN), que significa um valor prognóstico de, em caso o Teste Avaliativo de DTM ser positivo ou negativo, qual seria a probabilidade de a pessoa ter ou não ter efetivamente DTM.

O cálculo foi feito utilizando o Qui-Quadrado, tendo como referência para o VPP o percentual de testes positivos para o teste avaliativo em relação a todos os testes positivos; e para o VPN, o percentual de testes negativos para o teste avaliativo em relação a todos os testes negativos. Foi considerada significância estatística de $5 \%$ para todos os testes aplicados.

A reprodutibilidade foi analisada por meio da concordância entre os 3 examinadores de forma simultânea, por meio do Kappa de Fleiss e o Índice Kappa de Cohen, para análise 2×2. O Índice Kappa determina a proporção da concordância observada e esperada, considerando Kappa <0 (Pobre), 0-0,2 (Fraca), 0,21-0,4 (Razoável), 0,41-0,6 (Moderada), 0,61-0,8 (Substancial), >0,81 (Quase perfeita). ${ }^{14}$ 


\section{Resultados}

A amostra inicialmente contava com 13 indivíduos, sendo 3 excluídos por apresentarem traumatismo na região da face. A amostra final foi composta por 10 indivíduos. Os participantes apresentaram média de idade de 26 $\pm 2,6$ anos, cinco eram mulheres (50\%), quatro da raça branca (40\%), oito eram solteiros (80\%), cinco estudantes de fisioterapia (50\%), que consomem álcool e praticam atividade física com sete participantes (70\%) em cada item, conforme demonstrado na tabela 1.

Tabela 1. Características sociodemográficas da amostra estudada.

\begin{tabular}{ll}
\hline \multicolumn{1}{c}{ Variáveis } & $\mathbf{n ( \% )}$ \\
\hline Sexo & $5(50 \%)$ \\
Meminino & $5(50 \%)$ \\
Raça & \\
Branca & \\
Parda & $4(40 \%)$ \\
Negra & $3(30 \%)$ \\
Estado civil & $3(30 \%)$ \\
Solteiro & \\
Casado & $8(80 \%)$ \\
Divorciado & $1(10 \%)$ \\
Ocupação & $1(10 \%)$ \\
Estudante & \\
Fisioterapeuta & \\
Psicólogo & $5(50 \%)$ \\
Consumo de álcool & $4(40 \%)$ \\
\hline Sim & $1(70 \%)$ \\
\hline
\end{tabular}

Segundo o Índice Anamnésico de Fonseca, oito indivíduos foram diagnosticados com a presença de DTM, enquanto o Teste Avaliativo de DTM identificou nove participantes com presença desta disfunção.

No que diz respeito à acurácia diagnóstica, a porcentagem de indivíduos diagnosticados com a presença dessa disfunção, o que caracteriza a sensibilidade do Teste Avaliativo de DTM foi de 100\%, enquanto o valor de especificidade (ou porcentagem de indivíduos diagnosticados sem DTM) foi de 50\%. Já o Valor Preditivo Positivo foi de $88 \%$ e o Valor Preditivo Negativo foi de 50\%, conforme demonstrado na tabela 2. 
Tabela 2. Sensibilidade, Especificidade, VPP e VPN do Teste Avaliativo de DTM.

\begin{tabular}{|c|c|c|c|c|}
\hline & & \multicolumn{3}{|c|}{ ÍNDICE ANAMNÉSICO DE FONSECA } \\
\hline & & $\begin{array}{l}\text { Presença } \\
\text { de DTM }\end{array}$ & $\begin{array}{c}\text { Sem presença } \\
\text { de DTM }\end{array}$ & TOTAL \\
\hline \multirow{3}{*}{$\begin{array}{c}\text { TESTE AVALIATIVO DE } \\
\text { DTM }\end{array}$} & $\begin{array}{l}\text { Presença } \\
\text { de DTM }\end{array}$ & 8 & 1 & 9 \\
\hline & $\begin{array}{l}\text { Sem presença } \\
\text { de DTM }\end{array}$ & 0 & 1 & 1 \\
\hline & TOTAL & 8 & 2 & 10 \\
\hline
\end{tabular}

Sensibilidade $=8 / 8 \times 100=100 \%$

Especificidade $=1 / 2 \times 100=\mathbf{5 0} \%$

Valor Preditivo Positivo (VPP) $=8 / 9 \times 100=\mathbf{8 8} \%$

Valor Preditivo Negativo $(V P N)=1 / 1 \times 100=\mathbf{1 0 0} \%$

A análise de concordância geral entre os avaliadores o teste Kappa de Fleiss evidenciou que há reprodutibilidade razoável entre os resultados ( $K=0,26$ [IC 95\%: -0,099 - 0,617]; $z=1,420 ; p>0,05)$, porém não significante. Já o teste Kappa de Cohen mostrou que há reprodutibilidade pobre entre os avaliadores 1 e $2(K=-0,11 ; p>0,05 ;)$ e entre os avaliadores 1 e $3(K=-0,11 ; p>0,05 ;)$, e reprodutibilidade perfeita entre os avaliadores 2 e $3(K=1,00 ; p<0,05$;).

Figura 2. Acurácia inter-avaliadores.

\section{Concordância entre avaliadores}

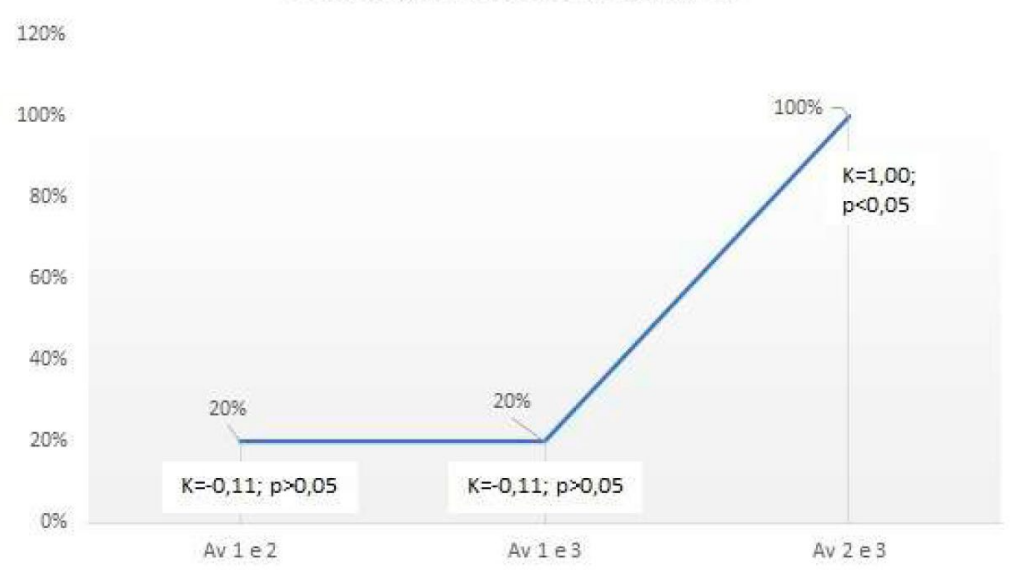

\section{Discussão}

A validação de instrumentos diagnósticos é necessária para gerar uma maior confiabilidade e reprodutibilidade destas ferramentas, o que corrobora com o objetivo de validação e reprodutibilidade desse estudo. Estudos de acurácia diagnóstica têm como objetivo validar testes que identifiquem de modo preciso os indivíduos afetados e os que não foram afetados por uma determinada disfunção.

Quando comparado ao IAF, o Teste Avaliativo de DTM obteve sensibilidade perfeita, o que significa que o TAvDTM é capaz de diagnosticar DTM nas pessoas que têm efetivamente DTM, sendo uma ótima ferramenta utilizada para triagem diagnóstica, por exemplo. No entanto, a especificidade do TAvDTM foi baixa, o que significa que o teste avaliativo pode diagnosticar DTM nas pessoas que efetivamente podem não apresentar a disfunção, o que se diz que seria um teste falso positivo. 
É importante salientar nesse momento que a diferença na especificidade entre os testes foi de um participante. Enquanto o IAF detectou dois participantes sem DTM, o TAVDTM detectou apenas um, gerando esse resultado de $50 \%$. Ou seja, essa proporção está superestimada e não pode, de fato, funcionar como medida real da especificidade do teste, necessitando de amostra maior para tal.

Semelhante ao analisado nesta pesquisa, em 2001 foi realizado um estudo para analisar o uso do Questionário para Triagem para Dor Orofacial e DTM, de maneira qualitativa e quantitativa, encontrando sensibilidade de $85.37 \%$ e especificidade de $80 \%$ para pacientes portadores de desordens musculares da região orofacial $(K a p p a=0,45)$ e baixa sensibilidade e especificidade para desordens intra-articulares (Kappa $=0,043) .{ }^{5}$ Apesar de utilizarem um instrumento que corresponde ao correto diagnóstico da disfunção temporomandibular, esses autores indicam a necessidade de uma avaliação multidisciplinar para pacientes que apresentassem sinais e sintomas específicos como: queixas de dores de cabeça, dores na face, dores na região auricular, pré-auricular e estalidos nas articulações. $\frac{5}{}$

Um estudo utilizou a curva Receiver operating characteristic (ROC) para determinar a acurácia de diagnóstiCo, sensibilidade e especificidade do IAF. Utilizando o escore total desse instrumento ( 0 a 100 pontos) como parâmetro de avaliação, encontraram uma alta acurácia diagnóstica no IAF para o diagnóstico de DTM, apresentando uma sensibilidade de $86,30 \%$ e uma especificidade de $91,19 \% .11$

Em 2018, autores sugeririam evitar o uso de analgésico para selecionar pacientes com DTM ou candidatos a intervenção, assim como sugerido no presente estudo como critérios de exclusão, e incluir testes clínicos no pescoço e na cintura escapular, levando, assim, uma análise e consideração de avaliação postural para o contexto do diagnóstico das disfunções temporomandibulares, afim de promover uma meIhoria no projeto de validação do RDC/TMD. ${ }^{15}$

O baixo resultado na reprodutibilidade do teste pode estar associado à habilidade na execução do teste por cada avaliador, já que existiu uma diferença de concordância entre os mesmos. Pode-se perceber que os avaliadores 2 e 3 concordam totalmente um com o outro nos achados, porém quando cada um foi comparado com o avaliador 1, foi encontrada diferença maior entre os resultados, o que parece sugerir que o avaliador 1 está discordando dos outros. Com isso, podemos inferir 2 possibilidades: o examinador 1 pode estar errando mais que os examinadores $2 \mathrm{e}$ 3 , ou os examinadores 2 e 3 , apesar de estarem concordando entre si, estão errando mais que o examinador 1.

Os resultados diferentes encontrados nos diagnósticos dos avaliadores podem ser explicados pelo fato do teste avaliativo de disfunção temporomandibular ser uma avaliação subjetiva e examinador-dependente, ou seja, cada pessoa vai realizar o teste de acordo com suas habilidades individuais e tendo como resultado o que ela conseguiu observar, e isso pode ter gerado a dificuldade dos três avaliadores em coincidir no diagnóstico.

Como o diagnóstico da disfunção temporomandibular pelo teste depende de o avaliador perceber ou não a presença de resistência durante a realização dos movimentos passivos propostos, outro motivo que poderia influenciar essa divergência entre os resultados pode ser o nível de treinamento de sensibilidade dos avaliadores para determinar uma possível resistência no corpo dos participantes.

Em um estudo realizado em 2009, os autores avaliaram a confiabilidade de um formulário para diagnóstico da severidade da disfunção temporomandibular, tendo encontrado resultados de Kappa entre 0,725 e 0,838 para as questões propostas. $\frac{16}{\mathrm{Em}}$ 2014, pesquisadores realizaram a validação e reprodutibilidade de um questionário de triagem para disfunção temporomandibular em adolescentes, encontrando valores de Kappa entre 0.529 e $0.884 .{ }^{17}$ Entretanto os dois estudos utilizaram como formas de avaliação questionário ou formulário e realizaram a validação intra-examinadores, enquanto esta pesquisa teve como instrumento um teste subjetivo de avaliação baseado em movimentos, e realizou a reprodutibilidade inter-examinadores. 
Na presente investigação, se faz necessário um maior tempo de treinamento para realização do teste, já que, devido à prática dos examinadores, há uma divergência entre os diagnósticos, com o Kappa variando entre pobre e perfeito. Em contrapartida, um estudo realizado em 2021, no qual foi realizada a validação e reprodutibilidade do Índice de Helkimo para o diagnóstico da disfunção temporomandibular, obteve reprodutibilidade inter-examinadores classificada entre moderada e substancial. ${ }^{18}$ Porém, apesar desse estudo apresentar um Kappa elevado, o Índice de Helkimo, além de não avaliar postura, foi validado para avaliação de severidade de sinais e sintomas, não fornecendo classificação diagnóstica. $\underline{9}$

Em um trabalho realizado em 2004, é dado ênfase à importância da avaliação da postura nos pacientes com DTM. A articulação temporomandibular é mantida em posição ortostática por meio de um mecanismo complexo, que envolve a musculatura da cabeça, pescoço e cintura escapular, em que qualquer alteração em sua conformação pode gerar alterações posturais, tanto nesses locais específicos quanto nas demais cadeias musculares. ${ }^{19}$ Sendo assim, em 2016 foi realizada a validação e reprodutibilidade de uma metodologia medindo a postura corporal e sua relação com a disfunção temporomandibular, avaliando desvios nos planos frontal e sagital, apresentando resultado inter-examinador excelente com Kappa > 0,87. 20 Entretanto, apesar de avaliar a associação entre DTM e postura, não é um estudo facilmente aplicável na prática clínica, já que foi realizada avaliação por meio de mensuração digital com uma metodologia específica criada para verificar a postura.

As limitações encontradas neste estudo podem estar relacionadas à conjuntura da pandemia do COVID-19, na qual o seguimento desse projeto passou por uma interrupção, levando à interferência no tamanho da amostra do estudo e no tempo de treinamento dos avaliadores. Por isso, os autores consideram o tamanho da amostra um fator contribuinte para um alto valor de sensibilidade e baixo valor da especificidade, necessitando, assim, de um número maior de indivíduos.

\section{Conclusão}

O Teste Avaliativo apresentou sensibilidade muito alta, sendo capaz de identificar todos os indivíduos com presença da DTM, podendo ser excelente ferramenta de triagem para DTM. Quanto à reprodutibilidade, esse estudo orienta sobre a necessidade de intenso e constante treinamento dos examinadores para a realização do teste avaliativo de DTM.

\section{Contribuições dos autores}

Neri YBO participou da concepção, delineamento, busca e análise estatística dos dados da pesquisa, coleta de dados, interpretação dos resultados, redação do artigo científico. Francisco ACS, Jesus IRN participaram da concepção e coleta de dados da pesquisa. Santos IEN participou da concepção, busca e análise estatística dos dados da pesquisa, coleta de dados, interpretação dos resultados, redação do artigo científico. Ferreira RS participou da concepção e do treinamento dos avaliadores. Góes ALB participou da concepção, delineamento, orientação e redação do artigo científico.

\section{Conflitos de interesses}

Nenhum conflito financeiro, legal ou político envolvendo terceiros (governo, empresas e fundações privadas, etc.) foi declarado para nenhum aspecto do trabalho submetido (incluindo, mas não se limitando a subvenções e financiamentos, participação em conselho consultivo, desenho de estudo, preparação de manuscrito, análise estatística, etc.).

\section{Referências}

1. Costa LM, Medeiros DL, Ries LG, Beretta A, Noronha MA. Avaliação das adaptações transculturais e propriedades de medida de questionários em língua portuguesa relacionados às desordens temporomandibulares: uma revisão sistemática. Fisioter Pesqui. 2014;21(2):107-12. https://doi.org/10.1590/18092950/35421022014

2. Nunes AM, Martinez EM, Lopes PRR, Bittencourt MAV, Canedo PMDM. Associação entre flexibilidade da cadeia muscular posterior e severidade de disfunção temporomandibular. Rev. Ciênc. Méd. Biol [Internet]. 2016;14(3):394. Disponível em: http:// repositorio.ufba.br/ri/handle/ri/23270 
3. Ferreira CLP, Silva MAMR, Felício CM. Sinais e sintomas de desordem temporomandibular em mulheres e homens. CoDAS. 2016;28(1):17-21. https://doi.org/10.1590/2317$\underline{1782 / 20162014218}$

4. Cantero LS, Morales, JDT, Espinosa IA, Rodríguez ET. Trastornos temporomandibulares en pacientes con maloclusiones. Rev Cubana Estomatol [Internet]. 2013;50(4):374-87. Disponível em: http://scielo.sld.cu/scielo.php?script=sci_arttext\&pid=S003475072013000400005\&lng=es

5.Manfredi APS, Silva AAD, Vendite LL. Avaliação da sensibilidade do questionário de triagem para dor orofacial e desordens temporomandibulares recomendado pela Academia Americana de Dor Orofacial. Rev Bras Otorrinolaringol. 2001;67(6):763-8. https://doi.org/10.1590/S0034-72992001000600003

6. Basso D, Corrêa E, Silva AM. Efeito da reeducação postural global no alinhamento corporal e nas condições clínicas de indivíduos com disfunção temporomandibular associada a desvios posturais. Fisioter Pesqui. 2010;17(1):63-8. https://doi. org/10.1590/S1809-29502010000100012

7. Azato FK, Castillo DB, Coelho TMK, Taciro C, Pereira PZ, Zomerfeld $\mathrm{V}$, et al. Influência do tratamento das desordens temporomandibulares na dor e na postura global. Rev. dor. 2013;14(4):280-3. https://doi.org/10.1590/S1806$\underline{00132013000400009}$

8. Horak FB. Postural orientation and equilibrium: what do we need to know about neural control of balance to prevent falls? Age Ageing. 2006;35 (Suppl 2):ii7-ii11. https://doi.org/10.1093/ ageing/afl077

9. Chaves TC, Oliveira AS, Grossi DB. Principais instrumentos para avaliação da disfunção temporomandibular, parte I: índices e questionários; uma contribuição para a prática clínica e de pesquisa. Fisioter Pesqui. 2008;15(1):92-100. https://doi. org/10.1590/S1809-29502008000100015

10. Cunha SC, Nogueira RVB, Duarte ÂP, Vasconcelos BCE, Almeida RAC. Analysis of helkimo and craniomandibular indexes for temporomandibular disorder diagnosis on rheumatoid arthritis patients. Braz J Otorhinolaryngol. 2007;73(1):19-26. https://doi.org/10.1016/s1808-8694(15)31117-4

11. Peña G, Díaz W, Flores G, Marinkovic K, Romo F, Schulz R. Concordancia entre los criterios diagnósticos RDC/TMD y su actualización DC/TMD, aplicados a la patología inflamatoria de la articulación temporomandibular. Rev. Clin. Periodoncia Implantol. Rehabil. 2019;12(2):70-3. http://dx.doi.org/10.4067/S0719$\underline{01072019000200070}$
12. Pastore GP, Goulart DR, Pastore PR, Prati AJ, Moraers M. Comparison of instruments used to select and classify patients with temporomandibular disorder. Acta Odontol. Latinoam. 2018;31(1):16-22. Citado em: PMID: 30056462.

13. Schwarzenbeck KCSB. Acurácia e correlação de instrumentos de avaliação da disfunção temporomandibular [dissertação] [Internet]. Campinas: Universidade Estadual de Campinas; 2013. Disponível em: http://bdtd.ibict.br/vufind/Record/CAMP d0053ab521d7fd7f764a09b9f1cb322f

14. Landis JR, Koch GG. The measurement of observer agreement for categorical data. Biometrics. 1977;33(1):159-74. Citado em: PMID: 843571

15. Steenks M, TürpJ, de Wijer A. Reliability and Validity of the Diagnostic Criteria for Temporomandibular Disorders Axis I in Clinical and Research Settings: A Critical Appraisal. J Oral Facial Pain Headache. 2018;32(1):7-18. https://doi.org/10.11607/ ofph.1704

16. Campos JADB, Gonçalves DAG, Camparis CM, Speciali JG. Confiabilidade de um formulário para diagnóstico da severidade da disfunção temporomandibular. Rev. bras. fisioter. 2009;13(1):843. https://doi.org/10.1590/S1413-35552009005000007

17. Franco-Micheloni AL, Fernandes G, Gonçalves DAG, Camparis CM. Temporomandibular disorders among Brazilian adolescents: reliability and validity of a screening questionnaire. J. Appl. Oral Sci. 2014;22(4):314-22. https://doi.org/10.1590/1678$\underline{775720130694}$

18. Alonso-Royo R, Sánchez-Torrelo CM, Ibáñez-Vera AJ, ZagalazAnula N, Castellote-Caballero Y, Obrero-Gaitán E, et al. Validity and Reliability of the Helkimo Clinical Dysfunction Index for the Diagnosis of Temporomandibular Disorders. Diagnostics (Basel). 2021;11(3):472. https://doi.org/10.3390/diagnostics11030472

19. Amantéa DV, Novaes AP, Campolongo GD, Barros TP. A importância da avaliação postural no paciente com disfunção da articulação temporomandibular. Acta Ortop Bras. 2004;12(3):1559. https://doi.org/10.1590/S1413-78522004000300004

20. Fernández RF, Carter $P$, Muñoz S, Silva $H$, Venegas GHO, Cantin $\mathrm{M}$, et al. Evaluation of validity and reliability of a methodology for measuring human postural attitude and its relation to temporomandibular joint disorders. Singapore Med J. 2016;57(4):204-8. https://doi.org/10.11622/smedj.2015159 